\title{
PENETRAZIONE DELLE RADIAZIONI LUMINOSE NEL GHIACCIO E NELLA NEVE
}

\author{
Silvio Polli
}

1. Comeralitio. - Il problema ha asemnlo recentemente notevole importanza per la neresilia, sentila specialmente dagli ishohiologi, di conosecere l'ambiente lumino-o nell'acequa di un lago sollo la copertura . insemale di ghiaceio e neve. Lat sua soluzione richiede pertanto la deleminazione dei corri-pondenti anflecienti di estinzione, distinti per gruppi di ratiazoni. Per l'acpua lali ricerele sono slate in parte gria enenule e in parle in rorso di esconzione.

I pochi lavori sull'aremento riguardano misure esemile con lermomelri. copple bermoelatriche, calorimetri e pireliometri, e pertanto

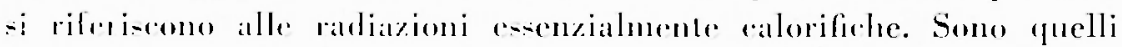

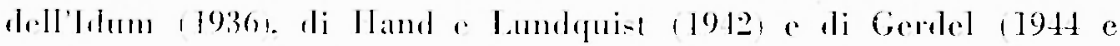

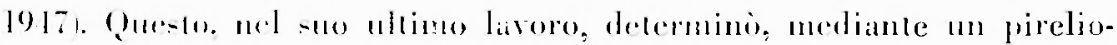
metro a lermoroppie di rame a contantana, i rapporti e i coeflicienti di e-linzione in fumzione della densila della neve, e mise in evidenza come l'anmento di gue-lat diminuineal il valore del roefliciente. Misure selellive per le radiasinni puramente luminuse non risultano ancora (a)enuile.

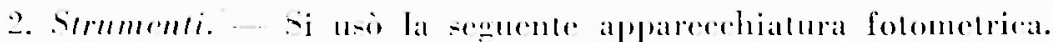

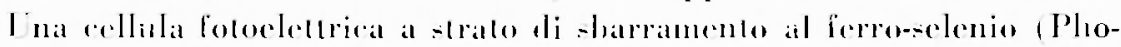

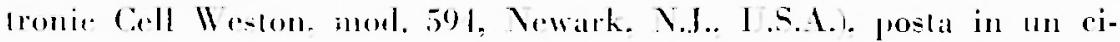

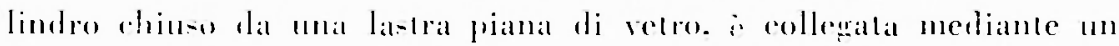
sisema di reistenze. con un walramomelro Kipp \& Konen di Moll, lipo "Mirmo \$-23n. Io strumento ha ma sensibilila massima di

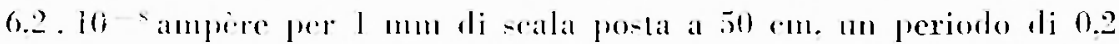

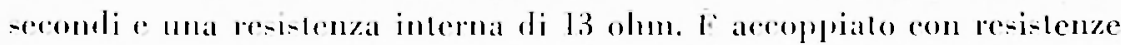
in serie e in derisazione in mode ehe quella del rircuito cellulat-gralvanonetro rimangal fluasi eostante sui 300 ohm quando si adoperano i vari shum per vasiare la sensihilita dello stumento.

Per mantenersi nei limiti di proporzionalita fra illuminazione della rellula e corrente elelleica sono sali aloperati. per illumina- 


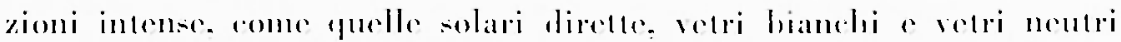
lella Calsa Zeins di lona.

3. Procedimento pratico. -- Messa la folemerlula orizzontale en esposta alla luee, si reselavano le resistenze el i veloi neulri in modo da spostare l'indiee luminomo del palvanometro vereo il fondo della

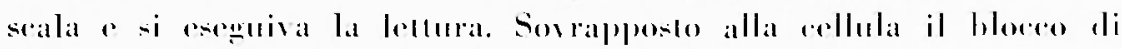

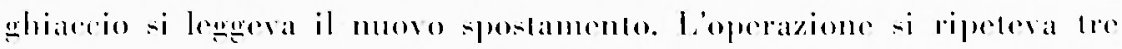

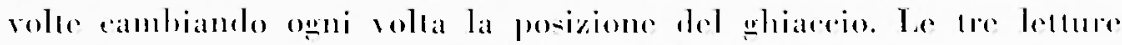
dillerisano di pochisimo lea di loro. le sariazoni enano inferiori all'te. Come misura effelliva si prendera la media delle tre. Lo stesso procerlimento reniva sepuito per ogni filtro coloralo rhe si sorrapponeva allat fotocellula.

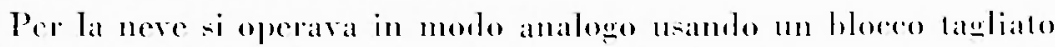
dal manto nevoso. Lo spessore degli strati cra misurato con molta

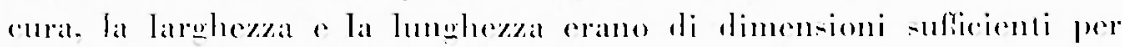
avere una diflusione laterale eselusivamente dovula al mezzo esaminato. Si pperi fra il ghiacrio e la nere, in riva al lago. nelle condizioni del lutlo naturali.

1. Procedimento teorico. - La relazione

$$
I=I_{n},-r^{-i s}
$$

$I_{\mathrm{n}}=$ intensili dei ragei penetranti nel mezo. $I=$ inten-ita degli stes-

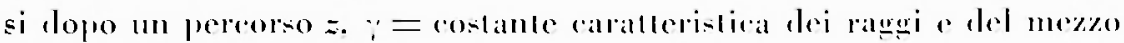

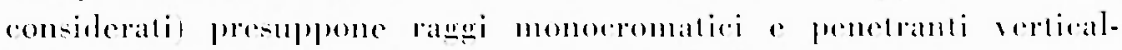
mente nello strato di ghiareio a neve. In pratiea no le ranliatoni sono

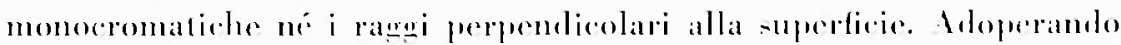
pere filtri selexionatori i pessibile operare in campi frentrali limilati e arvieinarsi rosi al cano leorico.

Per correwere wli effelli dellobliquita dei ragei oceore delerminare l'angolor ohe i rawei rifratti nel mezo fanno con la verticale c introdure quente valore nella formula precentente. rhe diviene:

$$
l=I_{0},-\cdots=\cdots,
$$

da cui. usamelo i logaritmi decimali, si olliene per $\because$ la formula:

$$
\because=2,30\left(\log I_{u}-\log I\right) \cos \quad r=.
$$

Medianle fuesta formula si calcolarome i coeflecienti di estinziome

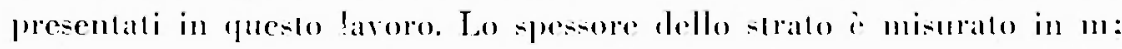




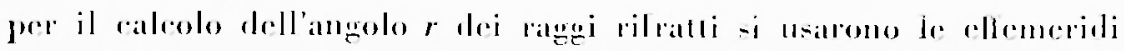

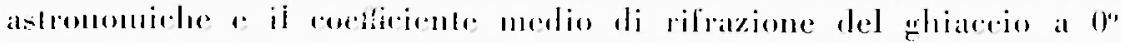

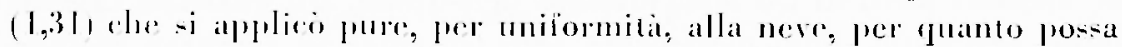
arere senso il significate di rifrazione nella neve.

Per rimatnere nel campe di validiti della formula si opere con

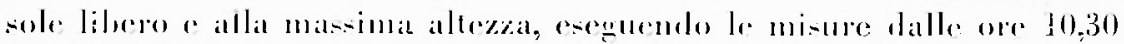

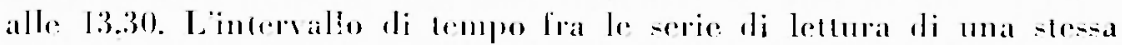
operazione risulta di qualele frazione di minuto. l'intensita luminosa patieamente non varia. Per i valori $I_{1)}$ e $I$ si allengome in queste costdizioni misme moluo precise.

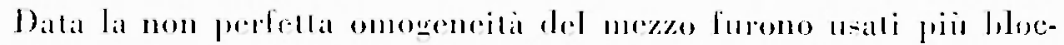

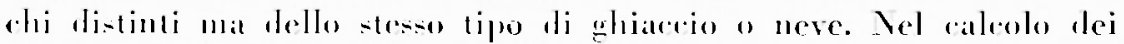

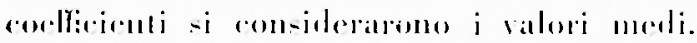

I vetri filtri arluperati somo quelli della Jenater Gla-werke Selote \& Gine gereisanente:

Uf; 2. violetlo estremo a parte drellultravioletto:

BG; 12. azzurero. tratratente per l'ultabioletto, non trasparente pere il roson a liultraromo:

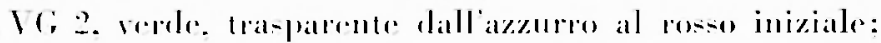

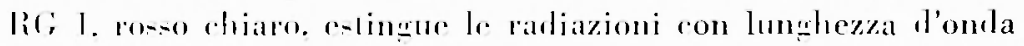
inferione al $594 \mathrm{~m} \mathrm{~m}$ :

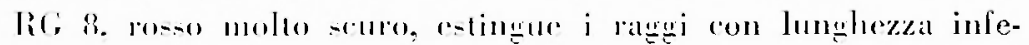

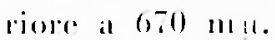

lo -pesole dei retri-filui i di 2 mun. Irame l'LG 2 che ne ha 1 min.

7. Le combizioni ambiemali ded momento. - Le misure per il

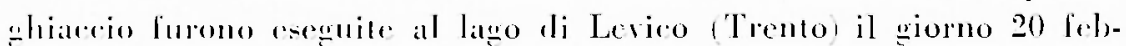

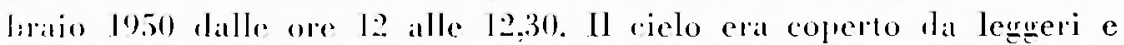

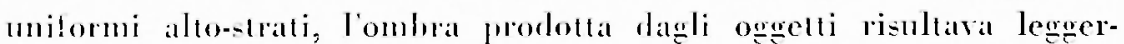
mente deloble. La temperatura dellaria, mi-urata con lo peicrometro

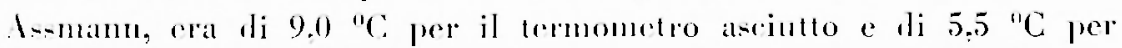

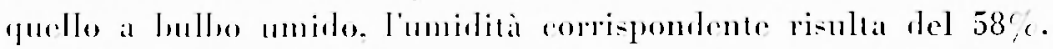

La temperatura follanequa sotto la copertura di ghiacedo risulto

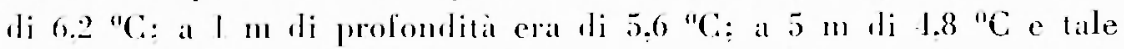
si mismor pure al fomlo 18 nul.

la) -trato di whiarcio roprival tutto il lago, con umo spessore di cima $8-10$ an, ad ara in lase di sgelo. La parte inferiore, in contatto

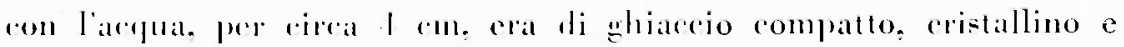

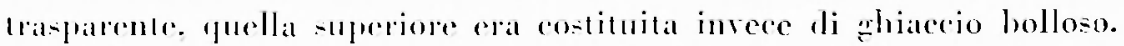


Le bollicine erano piò mumerose e grose verso la superficie, e areratno spessori medi di $2-3$ mm, massini di $5-6$ mm. L 11 qualsiasi ogegello posto nell'acqua inmediatamente sotlo il phiaceio risultava visibile in ogni suo particolare e colore. Buona duncue eral lat lrateparemal complessiva.

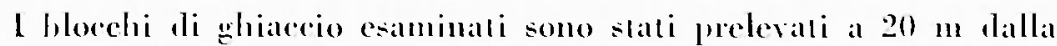
riva. Arevano spessori di 9 cm, erano langhi e lumghi cm 30, la loro temperatlura era di $0{ }^{\circ} \mathrm{C}$.

Le misure per la neve lurono eseguite alla riva del laggo di Caldonazzo (Trento) il giorno 22 dicembre 19/9, dalle ore 13 alle 11. Il cielo era coperto da leggeri e unilormi alo-strati. Lombra problota dagli oggelti risultava Ieggermente dehole. I utla la zonal eral coperta da 15-20 eu di neve cadula 10 giomi prima. Essa si presentana come

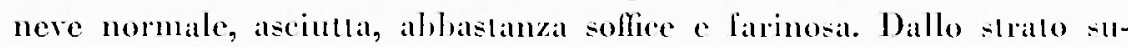
perficiale furono tagliali blocehi dello spesore di 8 am, lalloghi a lunghi 25 cm, la loro temperalura era di qualdehe decimo di grato sollo

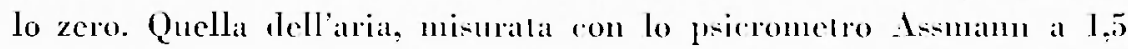
m sul suolo, era di 3,8 "C per il termometro asciutto e di 2,8 "C per quello a bulbo bagnato, l'umblita era dell'85'c. Laria cral calma, nel mallino si $i$ aruto un legerero vento da lisli.

6. I coefficienti di estinzione per il ghiurcio. -... Presentiamo i valori dei coelficienti $\gamma$ corriepondenti at gruppri di raldiazioni e alle comtizioni ㅃia indicate.

Coeficienti di estinzione $(1 / \mathrm{m})$ per il ghinceio.

\begin{tabular}{|c|c|c|c|c|c|}
\hline bianco & $\begin{array}{l}\text { viuletto } \\
\text { UG } 2\end{array}$ & $\begin{array}{l}\text { azzurro } \\
B C \quad 12\end{array}$ & $\begin{array}{l}\text { rerele } \\
16: 2\end{array}$ & $\begin{array}{c}\text { ronso ehe } \\
\text { he; l }\end{array}$ & 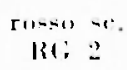 \\
\hline 1.52 & 4,63 & 2,28 & 1.25 & 373 & 188 \\
\hline
\end{tabular}

Soterole risulta l'azione selentiva del ghhiaceio sulla penetrazione dei singoli gruppi di rarliazioni. L'assorinimento is minimo per i rolori vereli, quasi doppio per gli azzurri, triplo per i rossi a poco meno che quadruplo per i violente i rossi seuri.

Conleontando questi valori con quelli corrispondenti all'acqua dello stesso laggo risulta che essi sono, come ordine di grrandezza, circa

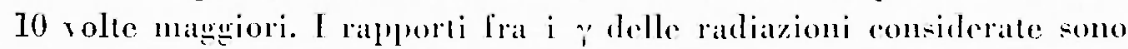
circal gli stessi nei due mezzi. cio vale a dire che l'arione selellival lel grhaceio differiace poeo da quella dell'alequa. 


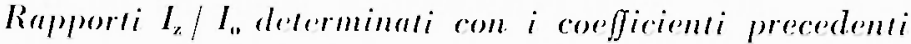
medianle la jormulu $I_{x}=I_{0} e^{-\gamma z}$

\begin{tabular}{|c|c|c|c|c|c|c|}
\hline $\begin{array}{c}\text { prolomil. } \\
\text { rm. }\end{array}$ & biamcon & $\begin{array}{l}\text { violello } \\
10: 2\end{array}$ & $\begin{array}{l}\text { :17xurro } \\
136 ; 1212\end{array}$ & $\begin{array}{l}\text { verde } \\
16 ; 2\end{array}$ & $\begin{array}{c}\text { messollo. } \\
\text { RO; } 1 .\end{array}$ & 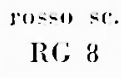 \\
\hline () & 100,0 & {$[000]$} & {$[(0), 0]$} & 1000 & 100,0 & 100,0 \\
\hline 2 & 97.5 & 91.2 & 95,5 & 97.5 & 92,8 & 90.7 \\
\hline 5 & 9111 & 79,3 & 89.2 & 91.0 & 83,0 & 78,3 \\
\hline 10 & 83,2 & 6.30 & 79,6 & 88,3 & 68,9 & 61,1 \\
\hline 20 & 77,8 & 34,7 & 6.31 & 77.8 & 17.1 & $3 \pi, 7$ \\
\hline 50 & 53.5 & 9.9 & 32,0 & 53,5 & 15,5 & 8.7 \\
\hline 100 & 28,6 & 0.98 & 102 & 236 & 2.1 & 076 \\
\hline 200 & 8,2 & 0.01 & 1.1 & 82 & 0,06 & 0.006 \\
\hline
\end{tabular}

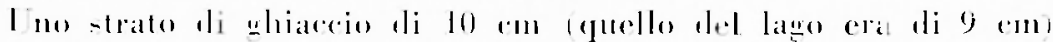
la-cial patare qua-i il 90 , delle ratiazioni veroli, il 60\%, delle vio-

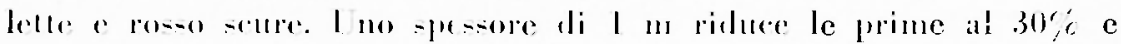
le seconde all'le. Deno 2 m prevalgono le verdi $(8 ; c)$ e le aroure

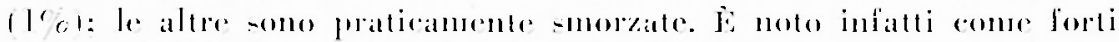

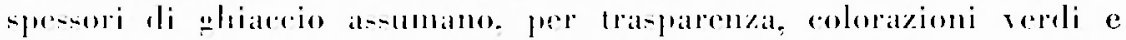
rroli-atz,110.

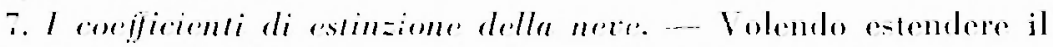

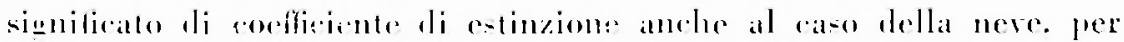
(fuantu in tale mezon la propatazione delle radiazioni puramente lu-

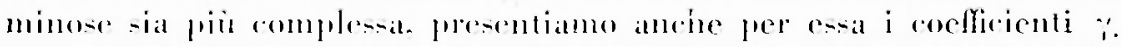
li-si rispereliande le eomelizioni del momento dello strato esmonato

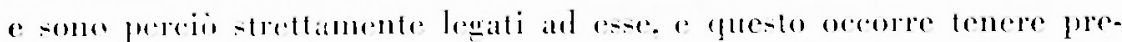

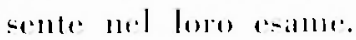

Coefficionti di estinzone $11 \mathrm{~m})$ per la neere.

\begin{tabular}{|c|c|c|c|c|c|}
\hline bi:anco & $\begin{array}{l}\text { vinlettu } \\
\text { lat } 2\end{array}$ & $\begin{array}{l}13 z u r r o \\
1 ; 6 ; 1 \geq 2\end{array}$ & $\begin{array}{l}\text { verde } \\
10 ; 2\end{array}$ & 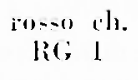 & $\begin{array}{c}r u s=0 \\
\text { Re } ; 2\end{array}$ \\
\hline 20.7 & 32.9 & 311 & 30.1 & 2195 & 29.7 \\
\hline
\end{tabular}

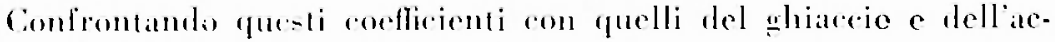
quat -i posomolare le segrenti oservazioni:

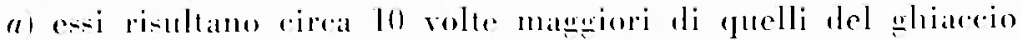
e lon vole magerori di quelli dell'arequa dello steso lago: 
b) l'alcqual. l'azione seleltiva della neve sulla penelrazione delle radiatzioni appitre dumpue molto debole:

c) nella neve, l'estinzione maggione si la per le radiazioni vio-

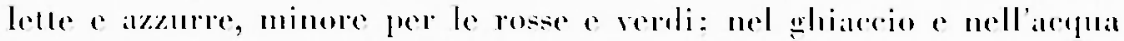

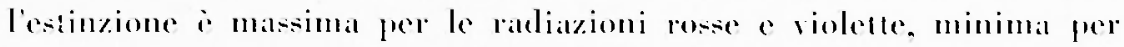
le rereli.

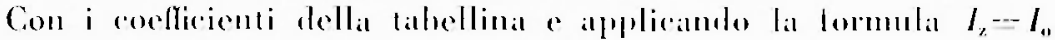

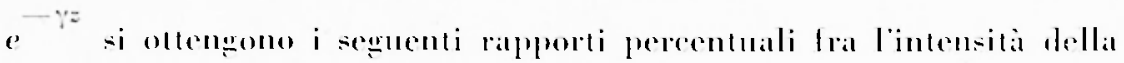
rarliavione incidente o quella allat profomblit z.

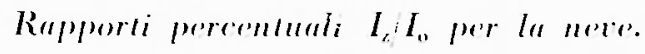

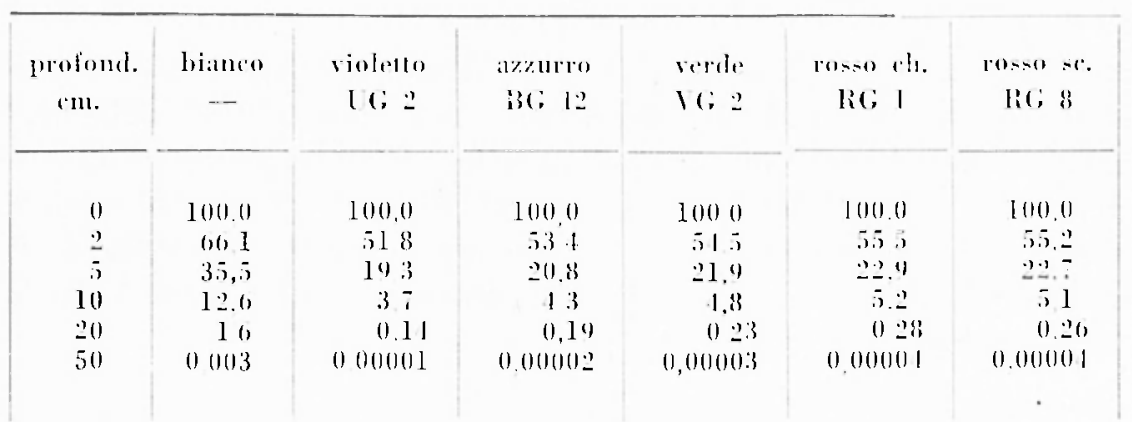

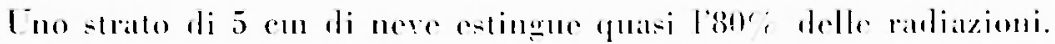

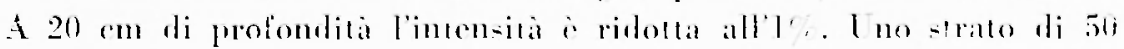

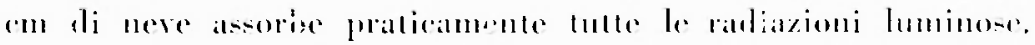

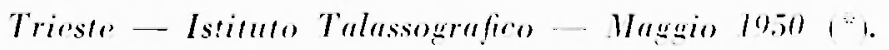

\section{RIASTI ITO}

Si determinano i corfficionti di estinsimen "i rapporti fra lo in-

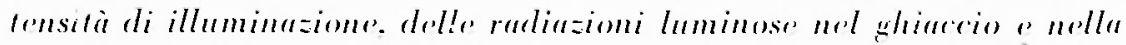
neer in condizioni nuturnli. Essi sono soleziomati per wruppi di rodiuzioni corrispondenti ai colori violello. azzurro. irrele. rosso chioro o

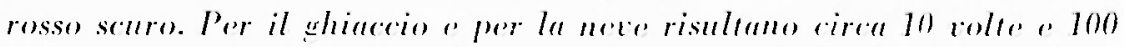
volle maggiori di quelli dellerequa.

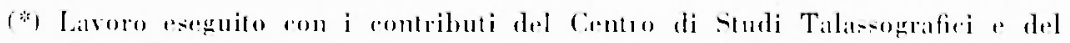
Centro di studi Mlpini del C.N.R. 


\section{BIBI,IOCHALIS}

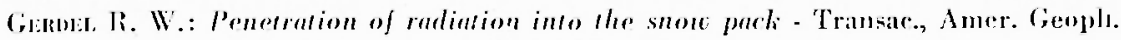
Inion., vol. 29 119181, n. 3, 361,-71.

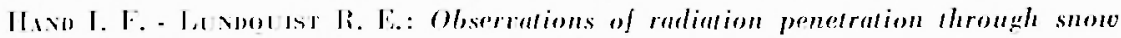
- Mon. Wea. Reve, wol. 71 (16/2), 22.25.

Int we S.: On the trismissibility of solur radimion of snow on ground - J. Met. Sor. Jilpiun., vol. II (1936), n. 2.

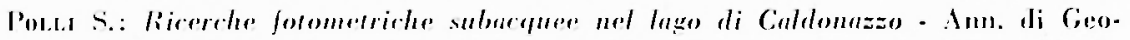
fisicil, sol. II (1919), II. I, patr. III-I?. 